\title{
AKARI view of star formation in NGC 1313
}

\author{
T. Suzuki ${ }^{1}$, H. Kaneda ${ }^{2}$, and T. Onaka ${ }^{3}$ \\ ${ }^{1}$ Institute of Space and Astronautical Science, Japan Aerospace Exploration Agency, 3-1-1 Yoshinodai, Chuo-ku, Sagamihara, \\ 252-5210 Kanagaawa, Japan \\ e-mail: suzuki@ir.isas.jaxa.jp \\ 2 Graduate School of Science, Nagoya University, Furu-cho, Chikusa-ku, 464-8602 Nagoya, Japan \\ 3 Department of Astronomy, Graduate School of Science, The University of Tokyo, 7-3-1 Hongo, Bunkyo-ku, 113-0033 Tokyo, Japan
}

Received 28 August 2012 / Accepted 18 February 2013

\section{ABSTRACT}

\begin{abstract}
Context. In the southwest region of NGC 1313, patchy star-forming regions are found. In the neighborhood, is a giant supershell with a diameter of $3.2 \mathrm{kpc}$. However, the direct association between star-forming regions and the giant supershell is still unclear.

Aims. We investigate the relation between the surface densities of the gas $\left(\Sigma_{\text {gas }}\right)$ and star formation rate $\left(\Sigma_{\mathrm{SFR}}\right)$ within the disk of NGC 1313 to obtain the spatial distribution of the star formation efficiency (SFE).

Methods. NGC 1313 was observed with the Infrared Camera (IRC) and Far-Infrared Surveyor (FIS) onboard AKARI in ten bands at 3.2, 4.1, 7, 11, 15, 24, 65, 90, 140, and $160 \mu \mathrm{m}$. With these AKARI ten-band images, we spectrally decomposed the stellar and polycyclic aromatic hydrocarbon (PAHs) components in addition to the cold $(\sim 20 \mathrm{~K})$ and warm $(\sim 60 \mathrm{~K})$ dust emission components. The cold and warm dust components were converted into the gas mass and the SFR.

Results. The SFE shows high values in the regions around the giant supershell, with the highest SFE in the galaxy in the southern spiral arm region. A similar trend can be seen in the spatial distribution of the PAH abundance relative to big grains. We find a powerlaw dependence of the star formation rate per unit area on the column density $\Sigma_{\mathrm{SFR}}$ proportional to $\Sigma_{\text {gas }}^{N}$. From a region-by-region analysis, the power-law index $N$ for the northern spiral arm is found to be $N \simeq 2.0$, which is observed on typical spiral arms, whereas those for the southern spiral arm and giant supershell regions have an index of $N \sim 1.6$.

Conclusions. The PAH abundance and the SFE show anomalously high values in regions around the giant supershell. The enhanced $\mathrm{PAH}$ abundance can be caused by shattering of big grains through slow shocks $\left(40 \mathrm{~km} \mathrm{~s}^{-1}\right)$. The power-law index $N=1.62 \pm 0.06$ obtained in the giant supershell region can be accounted for by the star formation scenario with collect-and-collapse in the expanding giant supershell. These pieces of evidence strongly indicate that the giant supershell has triggered the star formation and enhanced the SFE.
\end{abstract}

Key words. dust, extinction - stars: formation - galaxies: structure - galaxies: individual: NGC 1313 - infrared: ISM

\section{Introduction}

The evolution of galaxies remains a key subject in modern astrophysics. In particular, understanding the physical processes that control star formation in galactic disks is vital. The relation between the star formation rate (SFR) and the gas content is one of the most important subjects that allow insight into star formation processes in galaxies. Recent studies of this relation clearly show that interacting galaxies form their stars more efficiently than normal disk galaxies do (Genzel et al. 2010; Daddi et al. 2010b). This may arise from a different star-formation law. However, the underlying star formation mechanism is as yet unclear.

One possible mechanism is gravitational collapse of expanding neutral hydrogen shells, called supershells. Supershells are frequently seen in nearby starburst galaxies in addition to our Galaxy, the Magellanic Clouds, and nearby normal galaxies (e.g. Marlowe et al. 1995; Martin 1998; Heiles 1979, 1984; Dopita et al. 1985; Deul \& den Hartog 1990). Supershells with typical sizes of $\sim 100 \mathrm{pc}$ in diameter are usually thought to be formed by stellar feedback from OB stars. The accumulation of neutral hydrogen gas in shells is one means of generating the high densities and column densities required for the production of molecular gas. Thus, supershells have been suggested to be an efficient driver of forming molecular clouds and stars in them
(McCray \& Kafatos 1987). In particular, giant supershells (a few $\mathrm{kpc}$ in diameter) are considered to be caused by collisions of high-velocity gas clouds onto a gas disk or a single gamma ray burst (Tenorio-Tagle \& Bodenheimer 1988; Efremov et al. 1998). They can trigger the burst of star formation on a galactic scale. This galactic-scale star formation is expected to change the basic properties such as luminosity and chemical environment. Although evidence of supershell-associated star formation has been reported (e.g. Fukui et al. 1999), the star formation efficiency (SFE) of supershells is still observationally unclear.

Ryder et al. (1995) found a number of supershells in the face-on barred spiral galaxy NGC 1313. In particular, at the outer disk, there is a giant supershell of $3.2 \mathrm{kpc}$ in diameter, the largest among those in nearby galaxies. Figure 1 shows the center position of the giant supershell. Active star-forming regions in the southern spiral arm and southern satellite regions spatially correspond to local peaks in the H I column density of the giant supershell. The origin of the giant supershell is unclear. NGC 1313 is often described as a galaxy that shows intermediate features between Magellanic type and barred spiral galaxies (de Vaucouleurs 1963; Walsh \& Roy 1997). For example, its radial oxygen abundance distribution appears to be very uniform, with a mean value of $12+\log (\mathrm{O} / \mathrm{H})=8.4 \pm 0.1$ (Walsh \& Roy 1997), which is similar to what is observed in the 


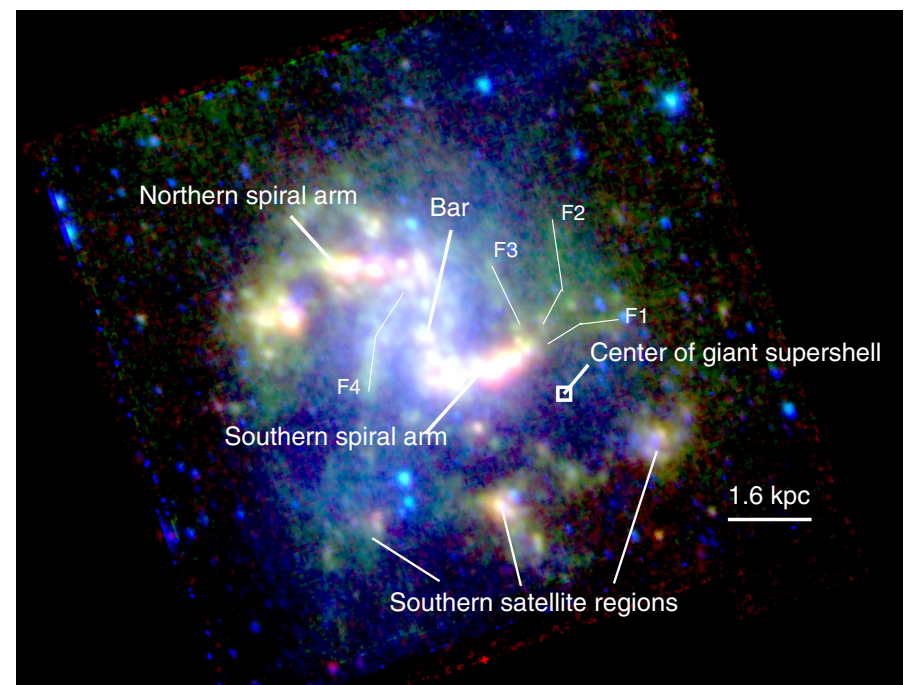

Fig. 1. Composite image of NGC 1313: AKARI $3.2 \mu \mathrm{m}$ (blue), $7 \mu \mathrm{m}$ (green), and $24 \mu \mathrm{m}$ (red). The center position of the giant supershell is marked as an open box (Ryder et al. 1995). Thin solid lines denoted by $\mathrm{F}_{1}-\mathrm{F}_{4}$ roughly trace the filament structures.

Large Magellanic Cloud (LMC). Because of the low-metallicity environment in $\mathrm{NGC} 1313,{ }^{12} \mathrm{CO}(J=1-0)$ line emission is faint and has only been detected at the galaxy center and close to the northern and southern spiral arms by the Swedish ESO Submillimeter Telescope (SEST; Bajaja et al. 1995; Contursi et al. 2002). Because the SFE is defined by the ratio of SFR per unit total $(\mathrm{HI}+\mathrm{H} 2)$ gas mass $M_{\text {gas }}$ : SFE $=S F R / M_{\text {gas }}$, the spatial distribution of the SFE in the disk of NGC 1313 has not been discussed so far.

Mid- and far-infrared (IR) observations provide an alternative method to investigate the SFE for these CO-faint objects. Spiral galaxies have warm and cold dust components in general, as first pointed out by de Jong et al. (1984) and subsequently confirmed by ISO observations (see the review of Sauvage et al. (2005) and references therein). The warm and cold dust components can be related to the SFR of massive stars and to the total gas content, respectively (Cox \& Mezger 1989). Moreover, midand far-IR observations have the advantage of least attenuation by dust extinction. Hence mid- and far-IR multi-band imaging observations provide us with an opportunity to investigate the SFE in individual regions of a galaxy by their dataset alone. To investigate the relation between SFR and gas surface densities within the disks of M81 and M101, Suzuki et al. (2010) applied the method on the AKARI/Far-Infrared Surveyor (FIS) datasets.

In this paper we report IR imaging observations of NGC 1313 performed with the Infrared Camera (IRC: Onaka et al. 2007) and the FIS (Kawada et al. 2007) onboard AKARI (Murakami et al. 2007), and discuss star-forming activities in the disk of NGC 1313. Our IRC data consist of six band images with central wavelengths of $3.2,4.1,7,11,15$, and $24 \mu \mathrm{m}$. Furthermore, the FIS data consist of four band images with central wavelengths of $65,90,140$, and $160 \mu \mathrm{m}$. The finer allocation of AKARI/IRC-FIS five bands $(24,65,90,140$, and $160 \mu \mathrm{m})$ enables us to spectrally decompose the warm and cold dust components. The spectral deconvolution analysis produces maps of the two components with a sufficiently high signal-to-noise ratio $(\mathrm{S} / \mathrm{N})$. Throughout this paper, we assume a distance of $4.1 \mathrm{Mpc}$ to NGC 1313 (Méndez et al. 2002).

\section{Observations and data reduction}

NGC 1313 was observed as part of the AKARI mission program "ISM in our Galaxy and Nearby Galaxies" (ISMGN; Kaneda et al. 2009) from December 2006 to June 2007. The observation $\log$ is listed in Table 1. Details of the IRC and FIS instruments and their in-orbit performance/calibration are described in Onaka et al. (2007), Kawada et al. (2007), and Shirahata et al. (2009).

The IRC observations were performed in the IRC02 observation mode with six bands: $N 3(3.2 \mu \mathrm{m}: 2.7-3.8 \mu \mathrm{m}), N 4(4.1 \mu \mathrm{m}$ : 3.6-5.3 $\mu \mathrm{m}), S 7(7 \mu \mathrm{m}: 5.9-8.4 \mu \mathrm{m}), S 11(11 \mu \mathrm{m}: 8.5-13.1 \mu \mathrm{m})$, $L 15$ (15 $\mu \mathrm{m}: 12.6-19.4 \mu \mathrm{m})$, and $L 24(24 \mu \mathrm{m}: 20.3-26.5 \mu \mathrm{m})$. Each field-of-view has a size of about $10^{\prime} \times 10^{\prime}$. The mid-IR images were created by using the IRC imaging pipeline software version 20071017. The background levels were estimated by averaging the values from multiple apertures placed around the galaxy without overlapping with extended emission, and were subtracted from the images. The bin sizes for the maps are 1". 4 for N3 and N4 bands and 2'. 3 for other bands. The FWHM of the point spread function (PSF) is $4^{\prime \prime}-7 "$ (Onaka et al. 2007).

The FIS was operated in photometry mode (FIS01) with the four band: N60 (65 $\mu \mathrm{m}$ : 50-80 $\mu \mathrm{m})$, WIDE-S $(90 \mu \mathrm{m}$ : 60-110 $\mu \mathrm{m})$, WIDE-L (140 $\mu \mathrm{m}: 110-180 \mu \mathrm{m})$, and N160 (160 $\mu \mathrm{m}: 140-180 \mu \mathrm{m})$. The four-band data were simultaneously obtained in a slow-scan observation for an area of about $10^{\prime} \times 20^{\prime}$ around the galaxy. The FIS data were processed with the AKARI official pipeline modules (version 20070914). Four-band images were created with grid sizes of 12 ". 5 for the WIDE-L and N160 bands, and 7'.5 for the WIDE-S and N60 bands. The background levels were estimated and subtracted using nearby regions of blank sky, which were observed in the beginning and at the end of the slow-scan observation. The FWHM of the PSFs are $\sim 45^{\prime \prime}$ for the WIDE-L and N160 bands and $~ 30^{\prime \prime}$ for the WIDE-S and N60 bands (Shirahata et al. 2009).

The IR flux densities at the ten bands were obtained by integrating the surface brightness within an aperture after subtracting the sky-background level. To obtain the fluxes of the whole galaxy, the photometric aperture with radius of 6 ' around the center was used, which roughly delineates the contour level of $\sim 0.5 \%$ of the peak intensity at the optical $B$ band. It includes most of the IR emission from the galaxy without degrading the $\mathrm{S} / \mathrm{N}$. The flux density errors for the AKARI/IRC bands are dominated by sky fluctuations, while those for the AKARI/FIS bands are dominated by systematic errors associated with both the detector artifacts and absolute calibration uncertainties.

\section{Results}

\subsection{Near- to mid-IR images}

The background-subtracted near- to mid-IR images of NGC 1313 obtained with the AKARI/IRC are shown in Fig. 2. The blank-sky level after background subtraction and its 1-sigma

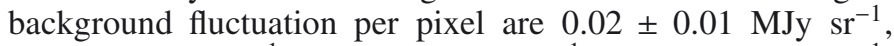
$0.01 \pm 0.01 \mathrm{MJy} \mathrm{sr}^{-1}, 0.02 \pm 0.02 \mathrm{MJy} \mathrm{sr}^{-1}, 0.04 \pm 0.03 \mathrm{MJy} \mathrm{sr}^{-1}$, $0.05 \pm 0.05 \mathrm{MJy} \mathrm{sr}^{-1}$, and $0.07 \pm 0.12 \mathrm{MJy} \mathrm{sr}^{-1}$ for N3, N4, S7, $S 11, L 15$, and L24.

Near-IR emission is dominated by photospheric emission from stellar populations. Figures $2 \mathrm{a}-\mathrm{b}$ clearly show the morphology of a barred spiral galaxy: the central bar and the two spiral arms. Emission from stars is the strongest at the galaxy center and prominent along the bar. We can also see an extended diffuse emission along the north-south direction that covers the 
Table 1. Observation log.

\begin{tabular}{lcccc}
\hline \hline Instrument & ${\mathrm{RA}(\mathrm{J} 2000.0)^{a}}$ & ${\text { Dec. }(\mathrm{J} 2000.0)^{a}}^{a}$ & Observation ID & Date \\
\hline IRC & 031816.05 & -662953.7 & $1400416-001$ & 2006 Dec. 03 \\
IRC & 031816.05 & -662953.7 & $1400417-001$ & 2006 Dec. 04 \\
FIS & 031816.05 & -662953.7 & $1402527-001$ & 2007 Jun. 03 \\
\hline
\end{tabular}

References. ${ }^{(a)}$ Units of right ascension are hours, minutes, and seconds, and units of declination are degrees, arcminutes, and arcseconds.
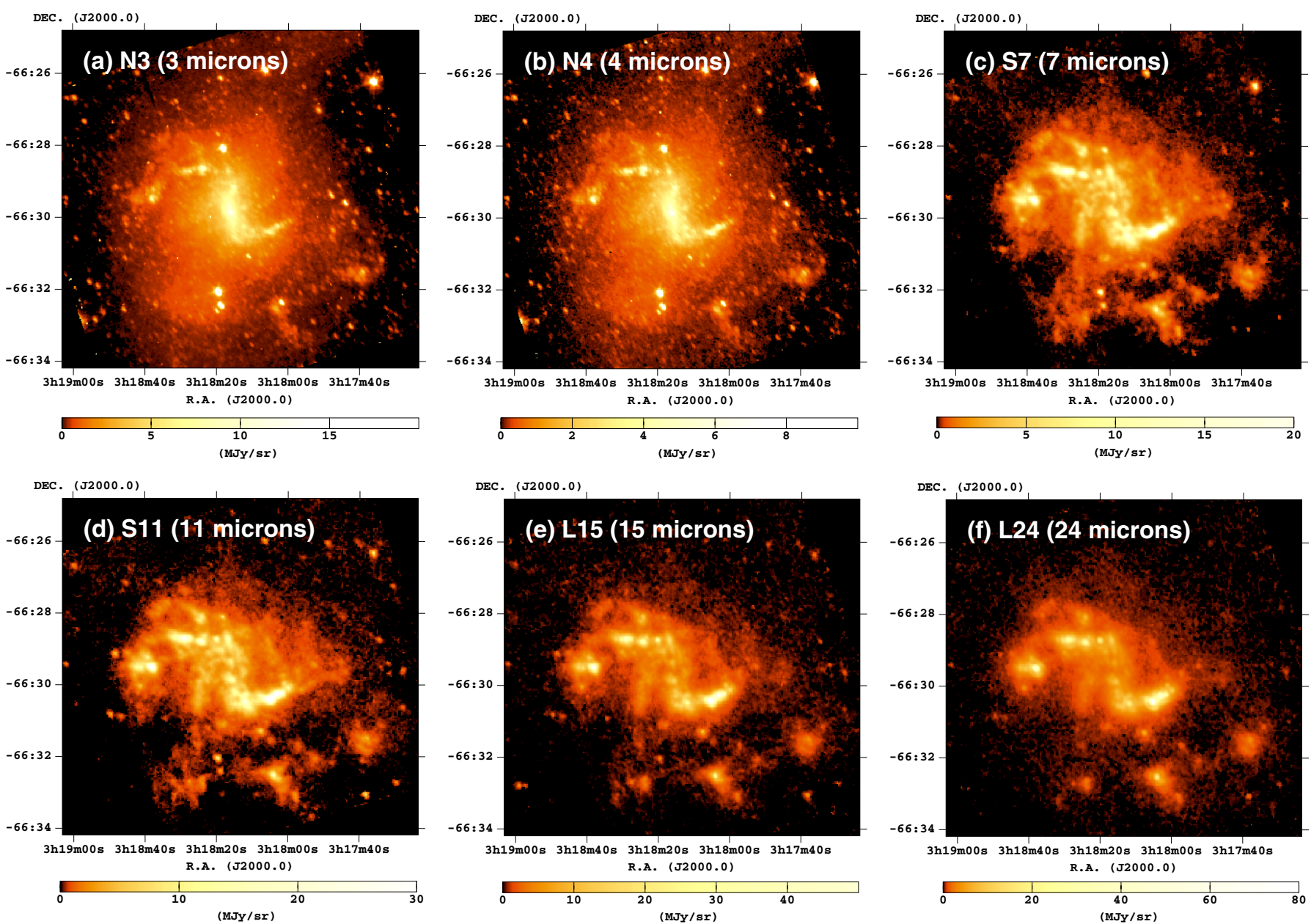

Fig. 2. Background-subtracted six-band images of NGC 1313 with the AKARI/IRC in the a) $N 3$; b) $N 4$; c) $S 7$; d) $S 11$; e) $L 15$; and f) $L 24$ bands at the central wavelengths of $3.2,4.1,7,11,15$, and $24 \mu \mathrm{m}$. The peak brightness is $2.4 \mathrm{MJy} \mathrm{sr}^{-1}(N 3), 1.4 \mathrm{MJy} \mathrm{sr}^{-1}(N 4), 12 \mathrm{MJy} \mathrm{sr}^{-1}(S 7), 21 \mathrm{MJy} \mathrm{sr}^{-1}$ (S11), $37 \mathrm{MJy} \mathrm{sr}^{-1}$ (L15), and $86 \mathrm{MJy} \mathrm{sr}^{-1}$ (L24).

whole galaxy, which is spatially coincident with optical emission (Ryder et al. 1995). Mid-IR emission is dominated by emission from very small grains (VSGs) and polycyclic aromatic hydrocarbons (PAHs). The $S 7$ and $S 11$ bands that include PAH emission features at $6.2,7.7,8.6$, and $11.3 \mu \mathrm{m}$ show bright emission along the two spiral arms, but faint emission at the galaxy center and the bar unlike the case seen in the N3 and N4 band images. Another striking feature in the two bands is the filamentary and patchy emission structure. As shown in Fig. 1, there are three large patches in the southwest of the disk, which are located in active star-forming regions called the southern satellite regions (Marcelin \& Gondoin 1983). Prominent filaments extend from the tip of the southern spiral arm $\left(F_{1}-F_{3}\right.$ in Fig. 1) and from the connection between the end of the bar and the northern spiral $\operatorname{arm}\left(\mathrm{F}_{4}\right)$, of which the filament $\mathrm{F}_{1}$ shows an arc-like emission structure around the giant supershell. The L15 and L24 band images mostly show emission from VSGs associated with massive star-forming regions. Star formation is more active in the two spiral arms and the satellite regions than in the bar.

\subsection{Far-IR images}

Figure 3 shows the FIS four-band images of NGC 1313 after background subtraction. The images are smoothed with boxcar kernels with a width of $15^{\prime \prime}$ for the N60 and WIDE-S bands and $25^{\prime \prime}$ for the WIDE-L and N160 bands for the display purpose. The blank-sky level after background subtraction and its 1-sigma background fluctuation per pixel are $0.01 \pm 0.50 \mathrm{MJy} \mathrm{sr}^{-1}$, $0.03 \pm 0.44 \mathrm{MJy} \mathrm{sr}^{-1}, 0.3 \pm 1.0 \mathrm{MJy} \mathrm{sr}^{-1}$, and $0.1 \pm 1.9 \mathrm{MJy} \mathrm{sr}^{-1}$ for N60, WIDE-S, WIDE-L, N160. The four-band images show bright spots in the two spiral arms and in the southern satellite regions. We found no local peak emission at the galaxy center in any band. The N60 and WIDE-S band images seem to show 

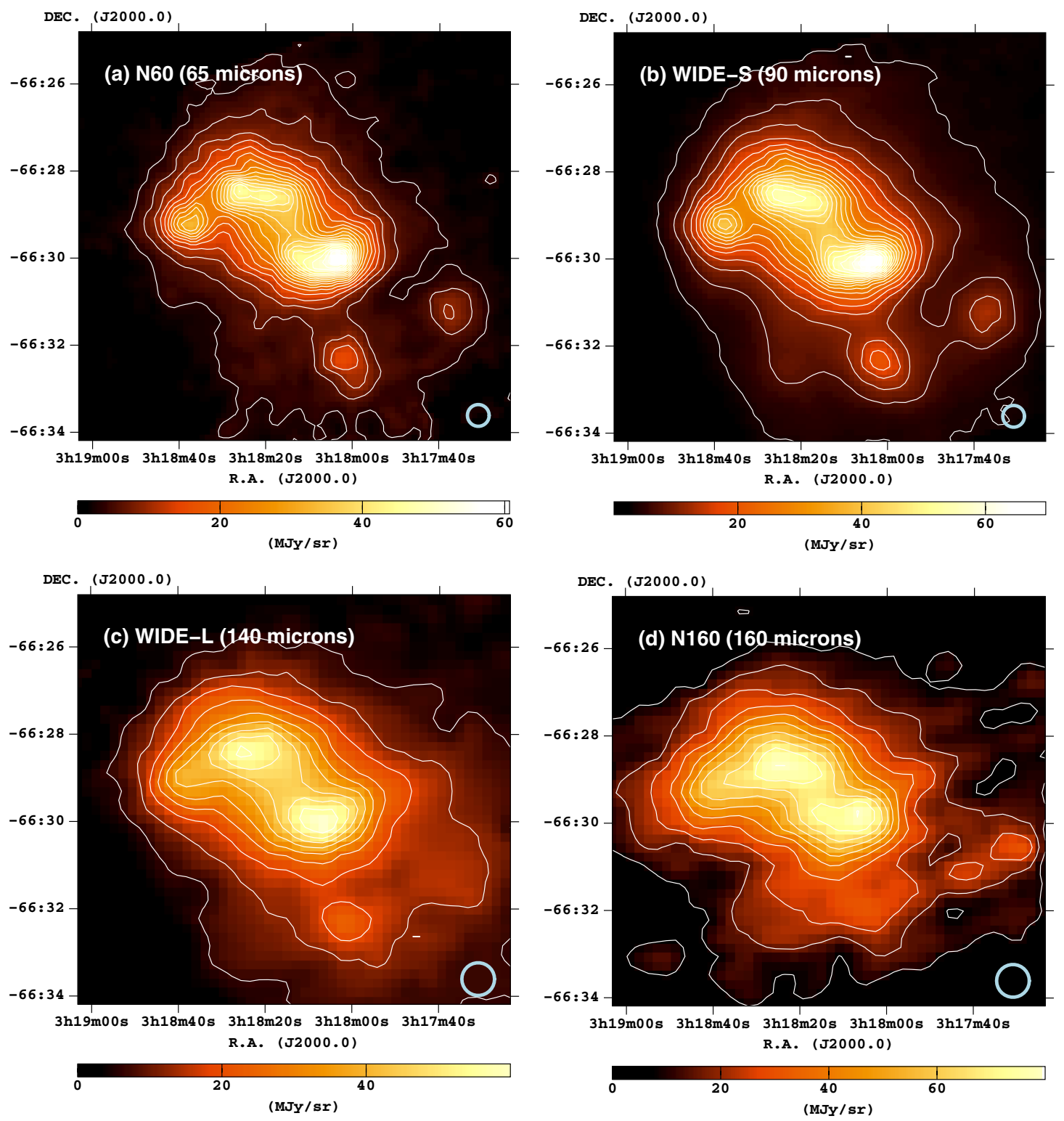

Fig. 3. Background-subtracted four-band images of NGC 1313 with the AKARI/FIS in the a) N60, b) WIDE-S, c) WIDE-L, and d) $N 160$ bands at the central wavelengths of $65,90,140$, and $160 \mu \mathrm{m}$. The peak brightness is $62 \mathrm{MJy} \mathrm{sr}^{-1}$ (N60), $72 \mathrm{MJy} \mathrm{sr}^{-1}$ (WIDE-S), $62 \mathrm{MJy} \mathrm{sr}^{-1}$ (WIDE-L), and $78 \mathrm{MJy} \mathrm{sr}^{-1}(N 160)$. The contours for the $N 60$ and WIDE-S bands are linearly spaced from 5\% of the peak brightness with a step of $5 \%$, while those for the WIDE-L and N160 bands are linearly spaced from 10\% of the peak brightness with a step of $10 \%$. In each image, the PSF size in FWHM is shown in the lower right-hand corner.

extended diffuse emission along the north-south direction, as seen in the N3 and N4 band images, while the WIDE-L and N160 band images seem to present the extended diffuse emission toward the east-west direction, as seen in the $S 7$ and $S 11$ band images.

\section{3. $\mathrm{H} \alpha$ image}

To investigate the relation between the warm dust and extinctioncorrected $\mathrm{H} \alpha$ line luminosities that are a combination of the observed $\mathrm{H} \alpha$ and $24 \mu \mathrm{m}$ IR luminosities (Calzetti et al. 2010), we used the continuum-subtracted $\mathrm{H} \alpha$ image taken from Hadfield $\&$ Crowther (2007). The data analysis and calibration processes are described in their paper. $[\mathrm{N} \mathrm{II}] / \mathrm{H} \alpha$ of 0.06 was applied to obtain pure $\mathrm{H} \alpha$ flux that was corrected for [N II] contamination (Hadfield \& Crowther 2007).

\subsection{Ultraviolet (UV) image}

To investigate the radiation field on a kiloparsec scale of NGC 1313, we used the GALEX image of NGC 1313. The far-UV (FUV) band image $\left(\lambda_{\text {eff }}=1539 \AA, \Delta \lambda=\right.$ $268 \AA$ ) was taken from the GALEX GR3 archive through the Multimission Archive at Space Telescope science institute (MAST). We retrieved the intensity and sky-background maps (filename: AISCHV4_393_39112). Correction for extinction toward NGC 1313, which includes foreground Galactic and internal extinction, was applied using the $E(B-V)$ values of 0.29 mag (Hadfield \& Crowther 2007) and the Cardelli et al. (1989) extinction law with $R_{V}=3.1$. By using the same aperture as that applied for the AKARI images, the FUV band surface brightness over the whole galaxy was estimated to be $(9.2 \pm 0.5) \times$ $10^{-4} \mathrm{Jy} \mathrm{sr}^{-1}$, or, equivalently $1.2 \times 10^{-6} \mathrm{erg} \mathrm{s}^{-1} \mathrm{~cm}^{-2} \AA^{-1} \mathrm{sr}^{-1}$. 
Table 2. Flux densities of NGC 1313 within an aperture radius of 6'.

\begin{tabular}{lc}
\hline \hline Band & $\begin{array}{c}\text { Flux density } \\
{[\mathrm{Jy}]}\end{array}$ \\
\hline$N 3(3.2 \mu \mathrm{m})$ & $0.70 \pm 0.06$ \\
$N 4(4.1 \mu \mathrm{m})$ & $0.45 \pm 0.06$ \\
$S 7(7 \mu \mathrm{m})$ & $1.0 \pm 0.2$ \\
$S 11(11 \mu \mathrm{m})$ & $1.2 \pm 0.3$ \\
$L 15(15 \mu \mathrm{m})$ & $0.7 \pm 0.2$ \\
$L 24(24 \mu \mathrm{m})$ & $4.1 \pm 1.2$ \\
$N 60(65 \mu \mathrm{m})$ & $48 \pm 10$ \\
$W I D E-S(90 \mu \mathrm{m})$ & $70 \pm 14$ \\
$W I D E-L(140 \mu \mathrm{m})$ & $116 \pm 35$ \\
$N 160(160 \mu \mathrm{m})$ & $108 \pm 43$ \\
\hline
\end{tabular}

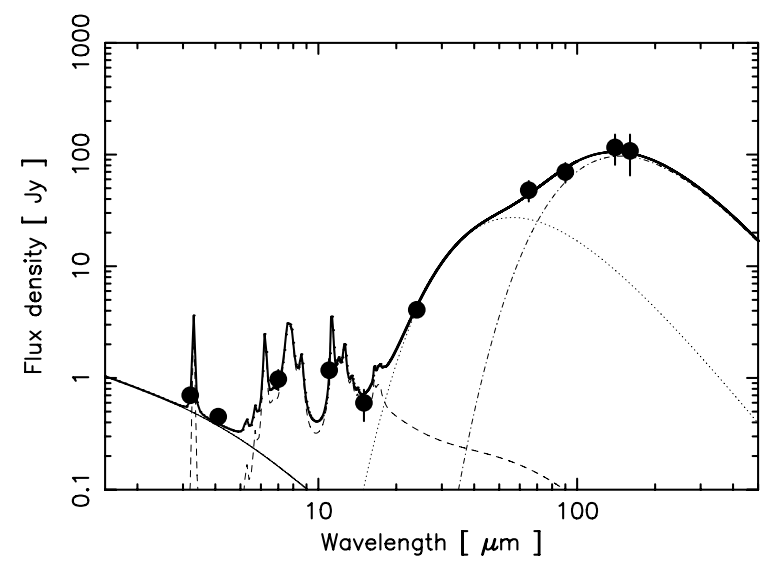

Fig. 4. Spectral energy distribution of NGC 1313, together with the best-fit two-temperature modified blackbody plus PAH and stellar component model. The filled circles correspond to the integrated flux densities in the ten AKARI bands. The thick solid line shows the best-fit model. We indicate the warm dust (dotted line), cold dust (dash-dotted line), PAH (dashed line), and stellar (thin solid line) components.

Considering that the FUV flux of the Habing field $\left(G_{0}=1\right)$, which is the integral of the flux over all directions, equals $2.3 \times$ $10^{-3} \mathrm{erg} \mathrm{s}^{-1} \mathrm{~cm}^{-2}$ at $\lambda=1530 \AA$ (Mathis et al. 1983), the observed FUV-band flux density within the aperture corresponds to $G_{0} \sim 2$ for the whole galaxy. On a kiloparsec scale, $G_{0}$ is estimated to range from $\sim 1$ to $\sim 10^{3}$.

\subsection{Spectral energy distribution of NGC 1313}

Figure 4 shows the spectral energy distribution (SED) of NGC 1313 constructed from the flux densities in Table 2. On the basis of the brightness profile along the scan direction, the loss of fluxes outside the aperture was estimated to be less than $8 \%$ (FIS bands) for the ten bands. The flux losses are negligible compared to other flux uncertainties. In a similar way as described in Suzuki et al. (2010), we fit the SED by a two-temperature modified blackbody with an emissivity power-law index of unity plus PAH and stellar component model. The PAH parameters were taken from Li \& Draine (2001) and Draine \& Li (2007) by adopting the PAH size distribution, fractional ionization, and a temperature probability distribution typical of diffuse ISM with the interstellar radiation field (ISRF) in the solar neighborhood. For the stellar component, we used the Mathis et al. (1983) stellar radiation field in the solar vicinity by applying a scaling factor.

The best-fit temperatures of cold and warm dust components are $T_{\mathrm{C}}=25 \pm 1 \mathrm{~K}$ and $T_{\mathrm{W}}=65 \pm 1 \mathrm{~K}$. The best-fit model provides estimates of the cold $\left(L_{\mathrm{C}}\right)$ and warm dust $\left(L_{\mathrm{W}}\right)$ luminosities in
Table 3. Properties of IR emission in NGC 1313.

\begin{tabular}{lcc}
\hline \hline Component & $\begin{array}{c}\text { IR luminosity } \\
{\left[L_{\odot}\right]}\end{array}$ & $\begin{array}{c}\text { Dust mass } \\
{\left[M_{\odot}\right]}\end{array}$ \\
\hline Cold dust & $9.6 \times 10^{8}$ & $1.0 \times 10^{6}$ \\
Warm dust & $1.3 \times 10^{9}$ & $1.2 \times 10^{4}$ \\
PAHs & $2.6 \times 10^{8}$ & - \\
Stars & $3.0 \times 10^{8}$ & - \\
\hline
\end{tabular}

the wavelength range of $3-1000 \mu \mathrm{m}$ and the PAH $\left(L_{\mathrm{PAH}}\right)$ and stellar $\left(L_{\mathrm{Star}}\right)$ luminosities in the wavelength range of $2-100 \mu \mathrm{m}$. Assuming that the far-IR emission is optically thin, the mass of dust $M_{\mathrm{d}}$ is estimated from the relation

$M_{\mathrm{d}}=10^{4}\left(\frac{L}{10^{8} L_{\odot}}\right)\left(\frac{T_{\mathrm{d}}}{40 \mathrm{~K}}\right)^{-5} M_{\odot}$,

where $L$ is the luminosity (Soifer et al. 1987). The dust temperatures were set to be equal to those derived from the above SED fitting. The derived luminosities and dust mass are summarized in Table 3 . The resulting total far-IR luminosity $L_{\text {FIR }}$ $\left(=L_{\mathrm{C}}+L_{\mathrm{W}}\right)$ and PAH luminosity are obtained as $2.3 \times 10^{9} L_{\odot}$ and $2.6 \times 10^{8} L_{\odot}$. Then, the ratio $L_{\mathrm{PAH}} / L_{\mathrm{FIR}}$ was estimated to be about 0.1 . By dividing the dust mass by the aperture area $\left(99 \operatorname{arcmin}^{2}\right)$, the dust mass surface density gives $1.0 \times 10^{4} M_{\odot}$ $\operatorname{arcmin}^{-2}\left(4.0 \times 10^{-3} M_{\odot} \mathrm{pc}^{-2}\right)$.

\subsection{Spatial distributions of stellar, $P A H$, warm dust, and cold dust components}

To derive the distributions of the components for NGC 1313, the spatial resolutions of the original IRC six-band, N60, and $W I D E-S$ images were reduced to match those of the WIDE-L and N160 images by convolving the former images with a Gaussian kernel. The images were then re-sized with the common spatial scale among the ten bands, 12 '. 5 pixel $^{-1}$. An individual SED constructed from the ten band fluxes at each image bin was then fitted with the same model as applied to the whole galaxy (see Sect. 3.5). Here the PAH parameters were again taken by assuming the ISRF in the solar neighborhood. The assumption does not affect the spectral shape for $G_{0} \lesssim 10^{3}$ (Draine \& Li 2007). In fact, $G_{0}$ on the same spatial scale ranges from $\sim 1$ to $\sim 10^{3}$ within the disk of NGC 1313 derived from the FUV image. For the local SED-fitting procedure, the temperatures of the two dust components and the amplitudes of the stellar, $\mathrm{PAH}$, and the two dust components were set to be free. To exclude image bins with a poor fit in terms of a $99 \%$ confidence level, we masked the image bins with values of $\chi_{v}^{2}$ higher than 3.3 with four degrees of freedom (i.e., ten data points and six free parameters).

Figure 5 shows the spatial distributions of the stellar, $\mathrm{PAH}$, cold dust, and warm dust components. The stellar component shows prominent emission in the bar, while the other three components show bright emission along the two spiral arms and patchy emission in the southern satellite regions, which spatially corresponds to active star-forming regions, as shown in Fig. 6a. As can be seen in Fig. 5d, the cold dust component overall extends toward the east-west direction of the disk, unlike the spatial distribution seen in the warm dust component. The extended emission spatially resembles that seen in the PAH component and H I gas (see Fig. 6b); in particular, the emission structure extending from the tip of the southern spiral arm seen in the PAH and cold dust components traces the arc-like structure seen in the H I map, which seems to lie at the northern rim of the giant supershell. 

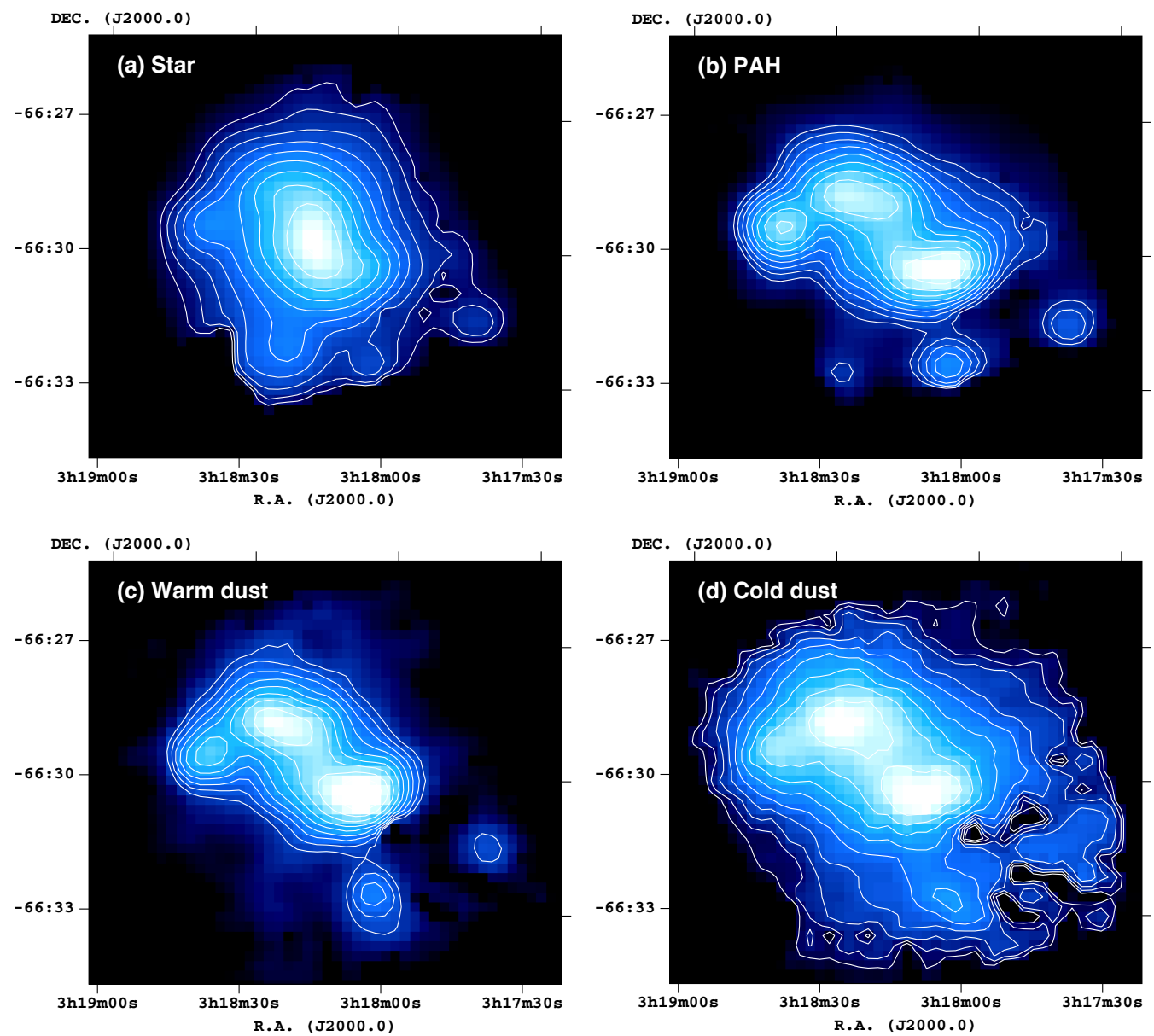

Fig. 5. Spatial distributions of the a) stellar, b) PAH, c) warm dust, and d) cold dust components of NGC 1313 . The contours are logarithmicaly spaced from $10 \%$ to $95 \%$ of the peak for the cold and warm dust components and from $5 \%$ to $95 \%$ of the peaks for the stellar, PAH components. The peak luminosities for the stellar, PAH, cold dust and warm dust components are $7.3 \times 10^{7}, 1.1 \times 10^{8}, 1.8 \times 10^{8}$, and $4.0 \times 10^{8} L_{\odot} \mathrm{kpc}^{-2}$.
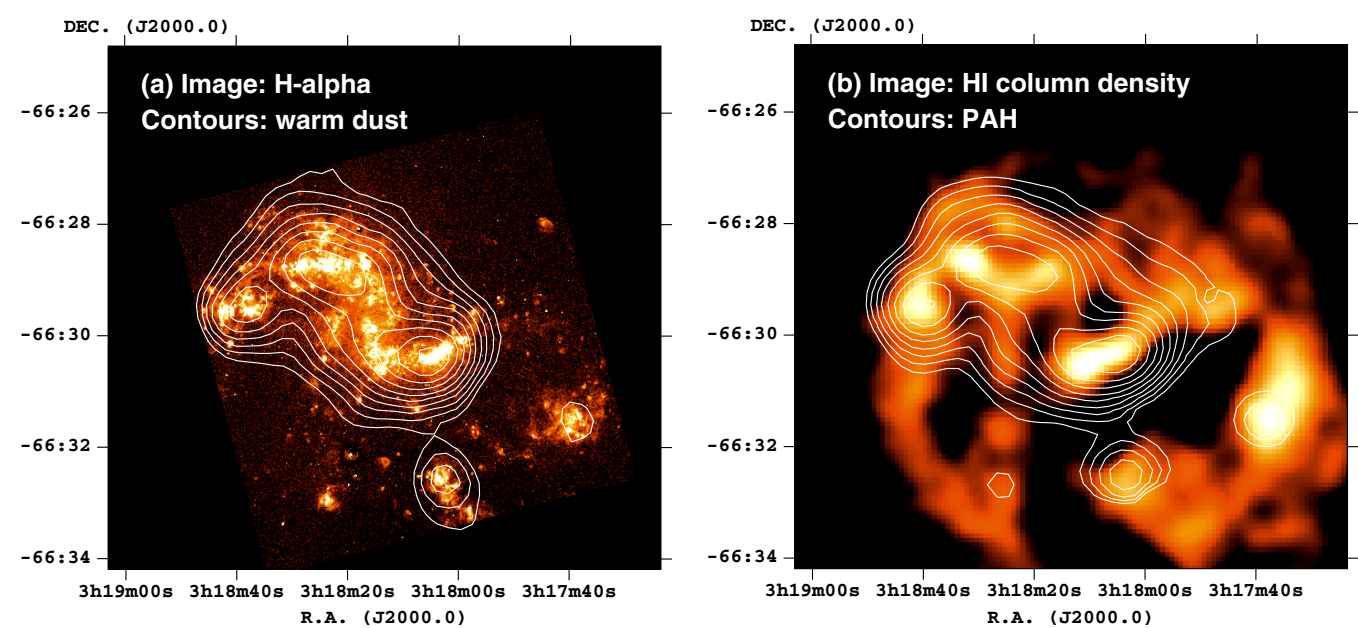

Fig. 6. Comparison with other wavelength images. a) H $\alpha$ image (Hadfield \& Crowther 2007) superposed on the warm dust contours. b) H I image taken from Ryder et al. (1995) superposed on the PAH contours. Contours for the warm dust and PAH components are the same as those in Figs. 5c and $b$.

\subsection{Spatial variations in relative $P A H$ abundance}

The map of $L_{\mathrm{PAH}} / L_{\mathrm{FIR}}$ was obtained to investigate the spatial variation in the relative PAH abundance. Figure 7 shows that the ratio in the southern spiral arm is higher than that in the northern spiral arm and is comparable to that at the eastern tip in the northern spiral arm. In addition, the map clearly shows bright spots and a filament that spatially correspond to the southern satellite regions and filament $F_{1}$ extending from the western tip in the southern spiral arm, respectively. In these regions with high values of $L_{\mathrm{PAH}} / L_{\mathrm{FIR}}$, the HI gas moves in a non-circular way with velocities higher than $10 \mathrm{~km} \mathrm{~s}^{-1}$ (Peters et al. 1994). 


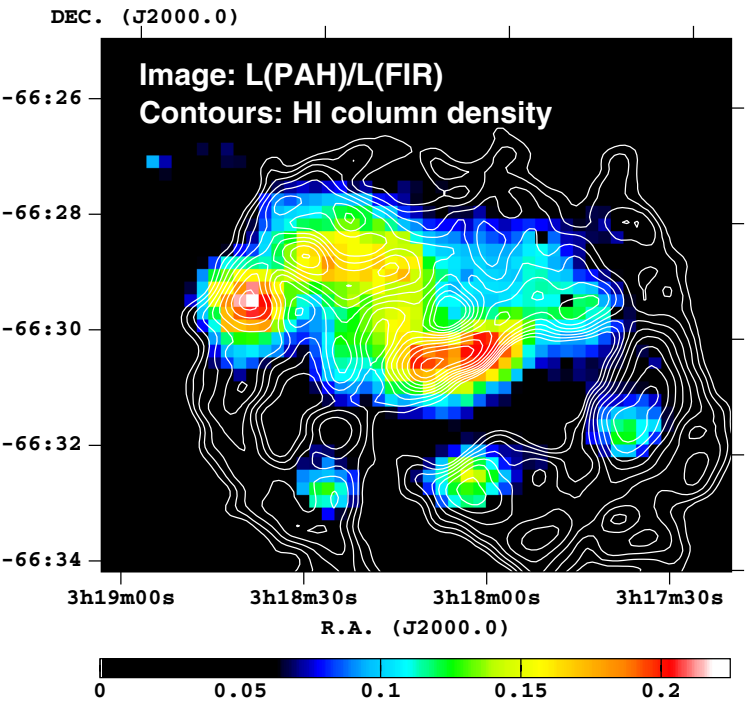

Fig. 7. Luminosity ratio of $L_{\mathrm{PAH}} / L_{\mathrm{FIR}}$. $L_{\mathrm{FIR}}$ is obtained from the sum of the cold and warm dust luminosities. The contours show the HI column density.

Therefore, it is possible that shock-excited $\mathrm{H}_{2}$ pure rotational lines significantly contribute to the IRC bands, which causes the anomalously high $L_{\mathrm{PAH}} / L_{\mathrm{FIR}}$. If this is the case, star formation in molecular gas associated with the shock-excited $\mathrm{H}_{2}$ is expected to be quenched (e.g. Cluver et al. 2010).

To investigate this possibility, we created the color-color diagram of S11/L15 versus S7/S11 for various positions in the disk of NGC 1313, as shown in Fig. 8. The diameters of the circles were set to be $\sim 20^{\prime \prime}$, which corresponds to a deprojected distance of $\sim 600 \mathrm{pc}$. The positions of the circles were selected from the area enclosed by the $3 \%$ level of the $24 \mu \mathrm{m}$ peak brightness at $\mathrm{H}$ II regions in the southern spiral arm. The green and yellow circles indicate fields in the northern and southern spiral arms. Figure 9 shows the resulting color-color diagram. In addition, the expected IRC colors only from $\mathrm{H}_{2}$ emission lines are plotted for both the power-law thermal (open boxes) and isothermal (open circles) $\mathrm{H}_{2}$ gas models and are the same as those in Fig. 7 of Shinn et al. (2009). In their calculations, $\mathrm{H}_{2}$ level populations are controlled by collisional excitation by $\mathrm{H}_{2}$ and $\mathrm{He}$ with the local thermal equilibrium (LTE). The ortho-to-para ratio of $\mathrm{H}_{2}$ was assumed to be 3.0. Shinn et al. (2009) adopted for the power-law distribution of the $\mathrm{H}_{2}$ gas temperature with its temperature range of 100 to $4000 \mathrm{~K}$ for the power-law thermal $\mathrm{H}_{2}$ gas model; $\mathrm{d} N \propto T^{-b} \mathrm{~d} T$, where $\mathrm{d} N$ is the infinitesimal $\mathrm{H}_{2}$ column density within the temperature range $(T, T+\mathrm{d} T)$. Observed color-color diagrams at the shock-cloud interactions where $\mathrm{H}_{2}$ emission lines are dominant agree well with those expected from the model (Shinn et al. 2009). For both model cases, the locus of the expected IRC colors moves from the lower left corner to the upper right corner as the $\mathrm{H}_{2}$ gas temperature increases. However, our data points are not distributed along any trajectories. In addition, we cannot find any difference in the diagram between the southern spiral arm and northern spiral arm regions. This suggests that the contribution of shock-excited $\mathrm{H}_{2}$ emission lines to $L_{\mathrm{PAH}}$ is negligible. Hence, $L_{\mathrm{PAH}} / L_{\mathrm{FIR}}$ traces the $\mathrm{PAH}$ abundance relative to big grains (BGs).

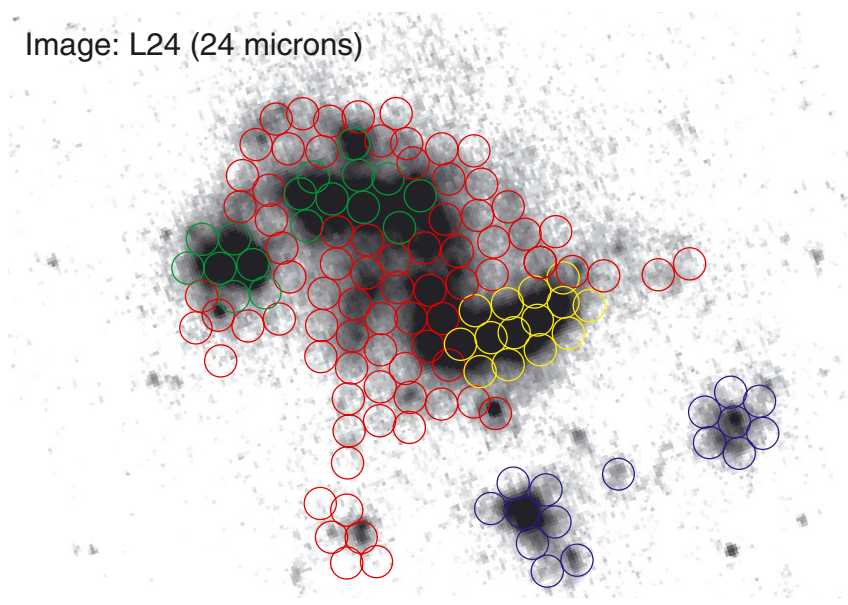

Fig. 8. Field positions in the disk of NGC 1313. The diameter of the circles is set to be $23^{\prime \prime}$. The green and yellow circles indicate regions in the northern and southern spiral arms. Regions surrounding the giant supershell are shown by the yellow and blue circles. The red circles indicate other region. The regions in the two spiral arms are defined as an area enclosed by a $3 \%$ level of the $24-\mu \mathrm{m}$ peak brightness.

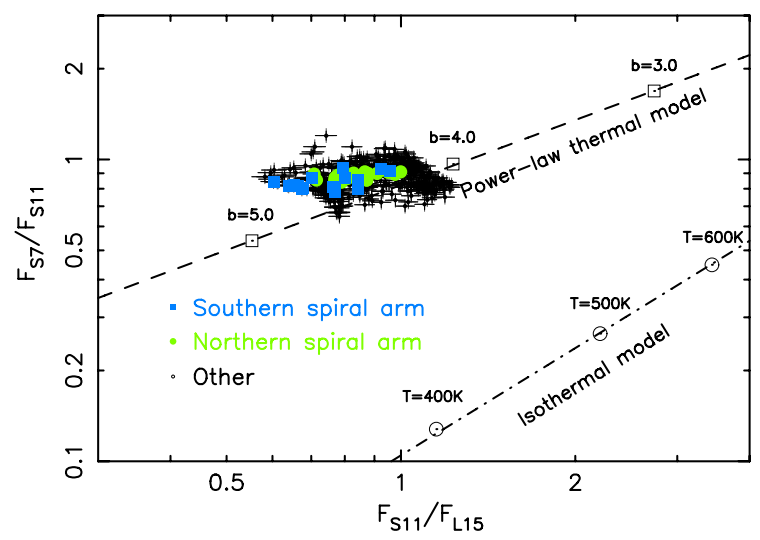

Fig. 9. IRC color-color diagram for the northern spiral arm (filled circles), southern spiral arm (filled boxes), and other regions (dots). Open boxes and open circles show expected colors of the pure $\mathrm{H}_{2}$ emission lines with the LTE case for both power-law thermal and isothermal $\mathrm{H}_{2}$ gas models(Shinn et al. 2009).

\section{Discussion}

\subsection{Relation between gas and SFR surface densities}

The cold dust component is heated by diffuse heating sources, such as non-ionizing UV photons that escape clearly from $\mathrm{H}$ II regions. The bright spots seen in the warm dust component are correlated with those seen in the $\mathrm{H} \alpha$ image (see Fig. 6a). As demonstrated by Suzuki et al. (2010), the cold and warm dust luminosity surface densities can be translated into the gas and SFR surface densities, respectively. We investigate the gas and SFR surface densities at various field positions in Fig. 8. The surface densities were then calculated by dividing gas mass $M_{\text {gas }}$ and SFR by the deprojected surface area.

To convert the cold dust luminosity surface density into the gas surface density at each field position, the local cold dust mass was estimated from Eq. (1) with the average temperature of the cold dust component derived from SED fitting within a circle. At low dust mass surface densities $\left(<\sim 0.1 M_{\odot} \mathrm{pc}^{-2}\right)$, Leroy et al. (2011) showed that the HI gas mass correlates well with the dust mass and dominates over $\mathrm{H}_{2}$ in the gas $\left(\mathrm{HI}+\mathrm{H}_{2}\right)$-to-dust 
mass ratio $\delta_{\mathrm{GDR}}$. As shown in Fig. 10, cold dust mass surface densities $\Sigma_{\mathrm{M}_{\mathrm{c}}}$ are distributed below $0.1 M_{\odot} \mathrm{pc}^{-2}$ and correlate with the H I gas mass surface densities $\Sigma_{\mathrm{HI}}$. In addition, as reported by Leroy et al. (2011), there is a diffuse H I component $\left(\Sigma_{\mathrm{HI}} \simeq 15 M_{\odot} \mathrm{pc}^{-2}\right)$, which is not associated with dust along the line of sight and thus can be obtained from the y-intercept of the dashed line in Fig. 10. Therefore, $\delta_{\mathrm{GDR}}$ for the field positions in the disk of NGC 1313 can be estimated from the H I gas-to-dust mass ratio after subtracting the diffuse $\mathrm{H}$ I component; $\delta_{\mathrm{GDR}}$ of 320 was obtained from the slope of the dashed line in Fig. 10. The gas mass was then derived from the mass of the cold dust $M_{\mathrm{C}}$ by using $\delta_{\mathrm{GDR}}$ as

$M_{\mathrm{gas}}(r, \theta)=\delta_{\mathrm{GDR}} M_{\mathrm{C}}(r, \theta)$.

The SFR was derived from the warm dust luminosity from Eq. (11) in Suzuki et al. (2010). The conversion is based on the relation between the warm dust and the extinction-corrected $\mathrm{H} \alpha$ line luminosities for positions in the disks of M101 and M81. The extinction-corrected $\mathrm{H} \alpha$ line luminosity $L(\mathrm{H} \alpha)_{\text {corr }}$ can be expressed as the combination of observed $\mathrm{H} \alpha\left(L\left(\mathrm{H} \alpha_{\text {obs }}\right)\right)$ and $24 \mu \mathrm{m}$ IR $(L(24 \mu \mathrm{m}))$ luminosities by applying the scaling factor $a_{24} \mu \mathrm{m}$ (Calzetti et al. 2007, 2010). Here, we investigate the relation in NGC 1313 to confirm the consistency of the relation obtained for M101 and M81. In Fig. 11, the open circles represent the relation for NGC 1313, while the open crosses and filled circles are those for M101 and M81 from Suzuki et al. (2010). These data overlap and show a clear correlation of the warm dust with $\mathrm{H} \alpha$ and $24 \mu \mathrm{m}$ IR luminosities. The result suggests that the relation for NGC 1313 is consistent with those for M101 and M81. However, open circles seem to level off around $L_{\mathrm{W}} \sim 10^{40.5} \mathrm{ergs} \mathrm{s}^{-1}$. The deviation of the NGC 1313 data from the dashed line suggests that either the $L\left(\mathrm{H} \alpha_{\text {obs }}\right)+L(24 \mu \mathrm{m})$ or $L_{\mathrm{W}}$ may have a problem. For the first possibility, $L(\mathrm{H} \alpha)_{\text {corr }}$ may be overestimated at luminous star-forming regions due to applying the constant value of $a_{24} \mu \mathrm{m}$. Therefore we investigated the relation between $a_{24 \mu \mathrm{m}}$ and $L(\mathrm{H} \alpha)_{\text {corr }}$ for 12 bright $\mathrm{H}$ II regions. Hadfield \& Crowther (2007) measured net $\mathrm{H} \alpha$ fluxes for the 12 bright HII regions in NGC 1313, whose positions are shown in Table 4. They estimated the extinction for individual H II region using the observed $\mathrm{H} \alpha / \mathrm{H} \beta$ line ratios; $L(\mathrm{H} \alpha)_{\text {corr }}$ shown in Table 4 is taken from Table 4 in Hadfield \& Crowther (2007). Then, we calculated the scaling factor for the $24-\mu \mathrm{m}$ IR luminosity $a_{24} \mu \mathrm{m}$ given by $\left(L(\mathrm{H} \alpha)_{\mathrm{corr}}-L(\mathrm{H} \alpha)_{\mathrm{obs}}\right) L(24 \mu \mathrm{m})^{-1}$, where $L(\mathrm{H} \alpha)_{\mathrm{obs}}$ and $L(24 \mu \mathrm{m})$ were estimated from the same positions and aperture sizes as those in Table 4 . The resulting relation for the 12 bright $\mathrm{H}$ II regions is shown in Fig. 12; $a_{24} \mu \mathrm{m}$ shows a decreasing trend and is not constant as $L(\mathrm{H} \alpha)_{\text {corr }}$ increases. At $L(\mathrm{H} \alpha)_{\text {corr }}$ of $\sim 10^{39} \mathrm{erg} \mathrm{s}^{-1}$, the value of $a_{24 \mu \mathrm{m}}$ is about half of the applied value, which means that the $L(\mathrm{H} \alpha)_{\text {corr }}$ derived from the combination of $\mathrm{H} \alpha$ and $24 \mu \mathrm{m}$ IR luminosities is overestimated by $\sim 40 \%$. As for $L_{\mathrm{W}}$, the SED fits of NGC 1313 data are good and do not indicate atypical SEDs. Accordingly there is no particular reason to suggest that the inference of $L_{\mathrm{W}}$ is problematic, although it cannot be ruled out. Thus, the trend of leveling off could be attributed to overcorrection of the extinction at luminous star-forming regions. From the dashed line in Fig. 11, which shows the best-fit linear model with a slope of unity for all data points, we obtain the following equation as

$\operatorname{SFR}\left(M_{\odot} \mathrm{yr}^{-1}\right)=5.5 \times 10^{-42} 10^{\left(\log L_{\mathrm{W}}\left(\mathrm{erg} \mathrm{s}^{-1}\right)-1.95\right)}$.

The parameters obtained from the best-fit linear model are the same as those in Eq. (11) in Suzuki et al. (2010) within the range of errors.

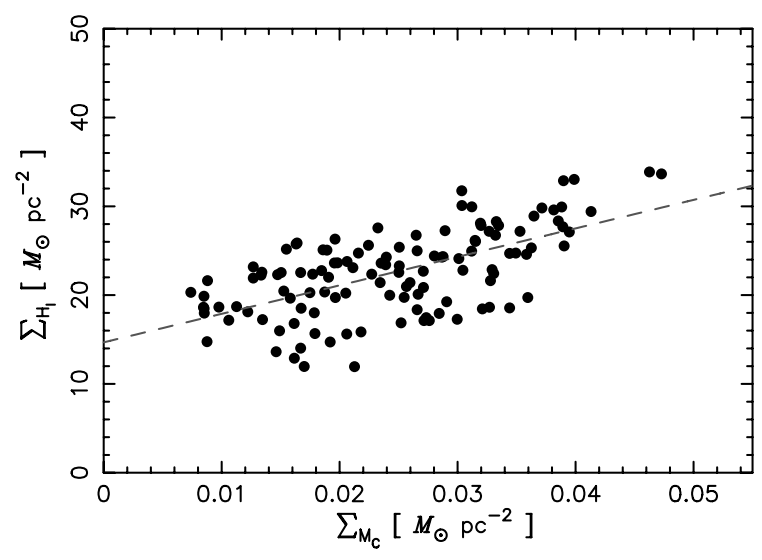

Fig. 10. Correlation between the H I gas and the cold dust mass surface densities for field positions in Fig 8. H I gas mass surface densities are obtained from H I column density map (Ryder et al. 1995). The dashed line shows the median H I gas mass-to-dust mass ratio of 320.

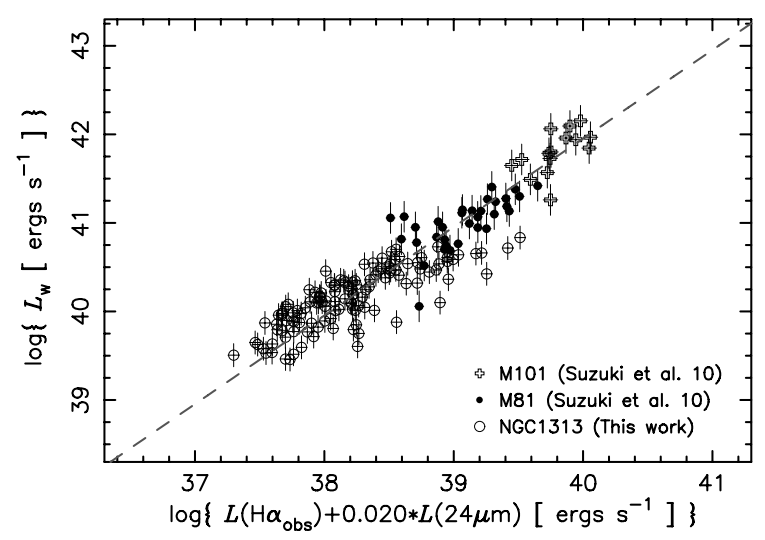

Fig. 11. Correlation between the warm dust luminosity and the combination of $\mathrm{H} \alpha$ and $24 \mu \mathrm{m}$ IR luminosities for NGC 1313 (open circles), M81 (filled circles) and M101 (open crosses). The dashed line shows the best-fit linear model with a unity slope for all data points for NGC 1313, M101, and M81. The data plots for M101 and M81 are taken from Suzuki et al. (2010). The scaling factor for the $24-\mu \mathrm{m}$ IR luminosity is taken from Calzetti et al. (2010).

From Eqs. (2) and (3), $L_{\mathrm{C}}$ and $L_{\mathrm{W}}$ can be converted into $M_{\text {gas }}$ and SFR, respectively. Uncertainties in both SFR and gas surface densities are dominated by errors in SED fitting. The average for the SFR surface densities is $(10 \pm 1) \times 10^{-3} M_{\odot} \mathrm{yr}^{-1} \mathrm{kpc}^{-2}$, whose error is estimated from the standard error of the mean. The average SFR surface density agrees well with that $(11 \times$ $10^{-3} M_{\odot} \mathrm{yr}^{-1} \mathrm{kpc}^{-2}$ ) inferred from the H $\alpha$ luminosity of SilvaVilla \& Larsen (2011). The global SFR within the aperture radius of $4.6 \mathrm{kpc}$ is estimated to be $0.65 \pm 0.07 M_{\odot} \mathrm{yr}^{-1}$, which also agrees with that for the same aperture size $\left(0.6 M_{\odot} \mathrm{yr}^{-1}\right.$, Hadfield \& Crowther 2007). In Fig. 13, the relations between gas $\left(\mathrm{HI}+\mathrm{H}_{2}\right)$ surface density $\left(\Sigma_{\text {gas }}\right)$ and SFR surface density $\left(\Sigma_{\mathrm{SFR}}\right)$ thus obtained for all field positions are overplotted as purple filled stars on the relations derived for individual galaxies shown in Fig. 2 of Daddi et al. (2010b). Our data become unreliable below $\Sigma_{\mathrm{SFR}}=10^{-3} M_{\odot} \mathrm{yr}^{-1} \mathrm{kpc}^{-2}$ ( $3 \sigma$ sensitivity). The solid and dash-dotted lines are the same as those in Daddi et al. (2010b); the solid line is a fit to the local spirals and the BzK galaxies, while the dash-dotted line is a fit to the local luminous infrared galaxies and submillimter galaxies (SMGs). Genzel et al. (2010); Daddi et al. (2010b) found that the powerlaw correlation (K-S law, $\Sigma_{\mathrm{SFR}} \propto \Sigma_{\text {gas }}^{N}$ ) for the individual galaxy 
Table 4. Positions and $L(\mathrm{H} \alpha)_{\text {corr }}$ for 12 bright $\mathrm{H}$ II regions.

\begin{tabular}{lcccc}
\hline \hline Region & ${\text { RA }(\mathrm{J} 2000.0)^{a}}^{a}$ & ${\text { Dec }(\mathrm{J} 2000.0)^{a}}$ & $\begin{array}{c}\text { Aperture diameter }^{b} \\
{[\operatorname{arcsec}]}\end{array}$ & $\begin{array}{c}L(\mathrm{H} \alpha)_{\text {corr }}{ }^{b} \\
\text { [erg s }^{-1} \text { ] }\end{array}$ \\
\hline 1 & 031823.9 & -662847.7 & 20 & $2.3 \times 10^{39}$ \\
2 & 031838.0 & -662919.7 & 12 & $4.3 \times 10^{38}$ \\
3 & 031837.8 & -662934.2 & 10 & $1.6 \times 10^{39}$ \\
4 & 031816.8 & -662844.3 & 15 & $8.5 \times 10^{38}$ \\
5 & 031805.3 & -663027.1 & 6 & $1.4 \times 10^{39}$ \\
6 & 031803.2 & -663016.5 & 24 & $2.3 \times 10^{39}$ \\
7 & 031803.5 & -663332.3 & 18 & $3.3 \times 10^{38}$ \\
8 & 031739.2 & -663126.5 & 18 & $4.1 \times 10^{38}$ \\
9 & 031818.3 & -662902.2 & 12 & $2.3 \times 10^{38}$ \\
10 & 031842.2 & -662932.2 & 12 & $6.9 \times 10^{38}$ \\
11 & 031819.6 & -662843.5 & 22 & $6.5 \times 10^{38}$ \\
12 & 031823.7 & -663255.9 & 20 & $3.7 \times 10^{38}$ \\
\hline
\end{tabular}

Notes. ${ }^{(a)}$ Units of right ascension are hours, minutes, and seconds, and units of declination are degrees, arcminutes, and arcseconds. ${ }^{(b)}$ Values are taken from Hadfield \& Crowther (2007).

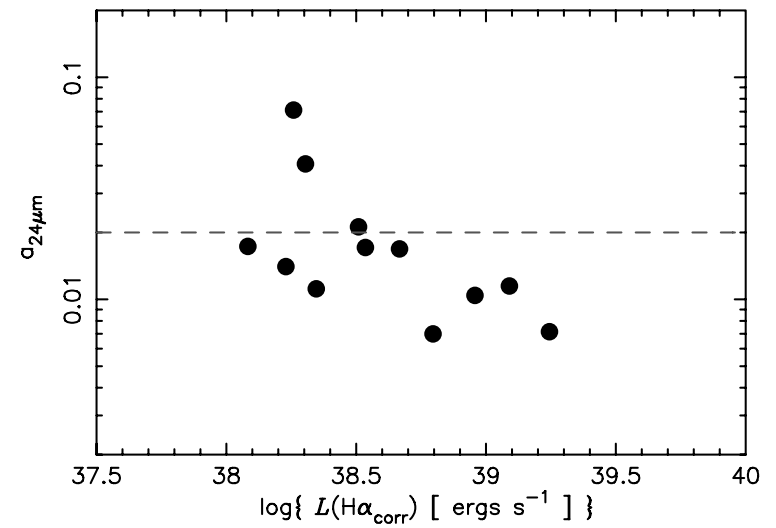

Fig. 12. Relation between $a_{24} \mu \mathrm{m}$ and $L(\mathrm{H} \alpha)_{\text {corr }}$ for 12 bright $\mathrm{H}$ II regions within NGC 1313. The dashed line shows the applied value of $a_{24} \mu \mathrm{m}$ in Fig. 11. $L(\mathrm{H} \alpha)_{\text {corr }}$ is taken from Table 4 in Hadfield \& Crowther (2007).

samples can be expressed not by a single sequence but by a double sequence: a bimodal star-formation law. Despite the difference in the spatial scale, the local relationship for the field positions shown in Fig. 8 agrees well with the global relationship for individual galaxies; in particular, we find that the K-S law for NGC 1313 is an intermediate state between normal and starburst galaxies. Next, as shown in Fig. 8, we focus on the K-S law in the disk of NGC 1313 by regions: (a) the giant supershell (yellow and blue circles); (b) the northern spiral arm (green circles); (c) the southern spiral arm (yellow circles); and (d) the others (red circles). To fit the data for each region with the K-S law model, as introduced in Blanc et al. (2009), we used the Monte Carlo (MC) approach combined with two-dimensional distribution comparison technique, which incorporates the intrinsic scatter in the K-S law as

$\Sigma_{\mathrm{SFR}}=A \Sigma_{\mathrm{Gas}}^{N} \times 10^{\mathcal{N}(0, \epsilon)}$,

where $A$ and $\mathcal{N}(0, \epsilon)$ are the normalization factor and the Gaussian distribution of the zero mean and the standard deviation $\epsilon$ that represents the intrinsic scatter from the power-law relation; free parameters are $A, N$, and $\epsilon$. For each set of the parameters, we generated $400 \mathrm{MC}$ realizations of the dataset $\left(\Sigma_{\mathrm{SFR}}, \Sigma_{\mathrm{Gas}}\right)$ taking account of the measurement uncertainties. To compare the distributions of points in $\Sigma_{\mathrm{SFR}}-\Sigma_{\mathrm{Gas}}$ plane, we then calculated the data point density in the grid resolution of

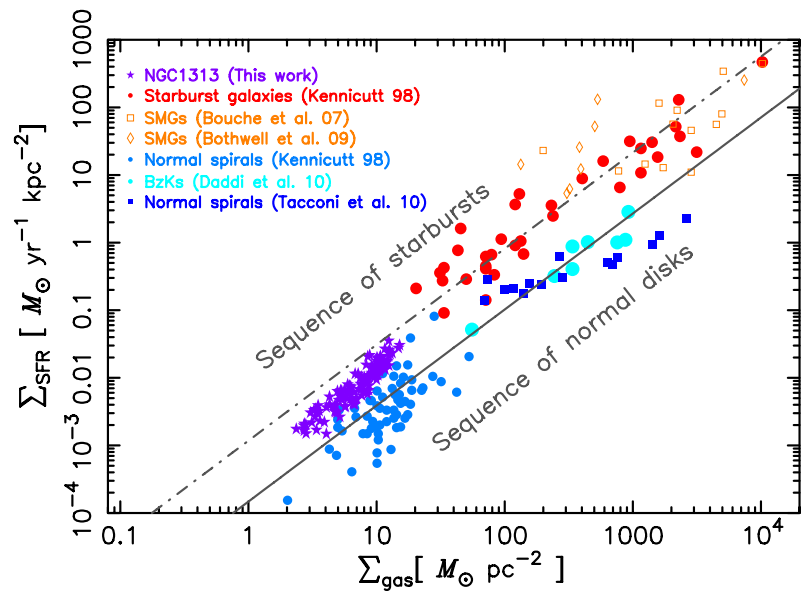

Fig. 13. Relation between gas surface density $\left(\Sigma_{\text {gas }}\right)$ and SFR surface density $\left(\Sigma_{\mathrm{SFR}}\right)$ within the disk of NGC 1313 (the purple filled stars) overplotted on the relation for individual galaxies in Fig. 2 of Daddi et al. (2010b). The red and light blue filled circles are infraredluminous starburst galaxies and normal spiral galaxies from the sample of Kennicutt (1998). The orange open diamonds and boxes are SMGs (Bouché et al. 2007; Bothwell et al. 2009). The blue filled boxes are $z=1-2.3$ normal galaxies (Tacconi et al. 2010). The cyan filled circles are the BzK galaxies (Daddi et al. 2010a). The solid and dashdotted lines are the same as those in Daddi et al. (2010b); the solid line shows a fit to normal spirals and BzKs galaxies with the K-S law model $(N=1.42)$, while the dash-dotted line shows the same relation shifted up by 0.9 dex to fit starburst galaxies and SMGs.

$\Delta \Sigma_{\mathrm{SFR}}=0.003 M_{\odot} \mathrm{yr}^{-1} \mathrm{kpc}^{-2}$ and $\Delta \Sigma_{\mathrm{Gas}}=2 M_{\odot} \mathrm{pc}^{-2}$ and compared the density between the models and the observations to derive the best-fit parameters and their standard deviations. Figures 14 and 15 show the observed K-S law together with the $400 \mathrm{MC}$ realizations of the data for the best-fit parameter set for each region and together with the best-fit power law from the MC method. The resulting power-law indices show various values from region to region as summarized in Table 5; the results may be associated with the difference in physical process of star formation. According to Suzuki et al. (2010), the power-law indices for spiral arms in M81 and M101 show $N \simeq 2.0$, which can be accounted for by star formation by cloud-cloud collisions enhanced in spiral density wave. The power-law index, $N \simeq 2.0$, obtained in the northern spiral arm may indicate the same star formation process. However, as for the southern spiral arm and 


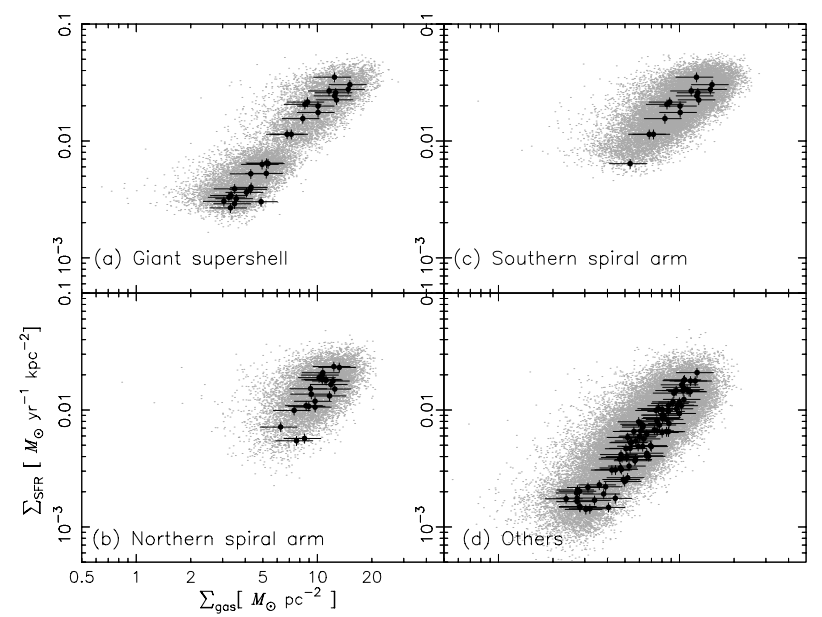

Fig. 14. Observed $\Sigma_{\text {gas }}-\Sigma_{\text {SFR }}$ relation for each region (black dot) together with the $400 \mathrm{MC}$ realizations of the data for the best-fit parameters (gray dots).

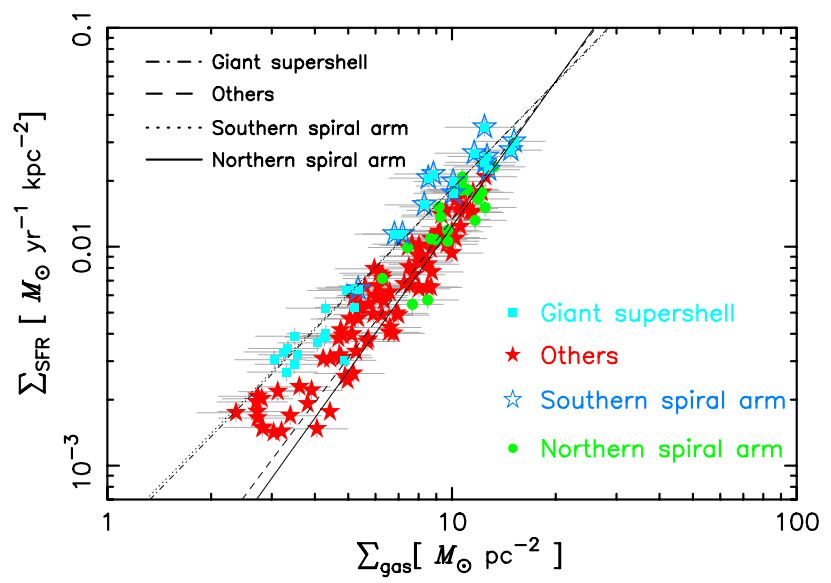

Fig. 15. $\Sigma_{\text {gas }}-\Sigma_{\text {SFR }}$ relation for various regions in NGC 1313. The cyan filled boxes, blue open stars, green filled circles, and red filled stars represent the giant supershell, southern spiral arm, northern spiral arm, and other regions. The lines are the best-fit power law from the MC method for the giant supershell (dash-doted line), southern spiral arm (dotted line), northern spiral arm (solid line), and others (dashed line).

the giant supershell, the slope $(N \sim 1.6)$ is less steep than that for the northern spiral arm. One of the possible star-forming processes to account for $N \sim 1.6$ seems to be a self-gravitational mechanism (Elmegreen 1994b).

\subsection{Enhanced star-forming activity in regions around the giant supershell}

To compile the map of the SFE within the disk of NGC 1313, the SFE was calculated by dividing the SFR by $M_{\text {gas }}$ on a pixel to pixel basis. We masked the pixels with a poor fit in terms of a $99 \%$ confidence level for SED fitting (see Sect. 3.6). In Fig. 16, the SFE clearly shows the spatial variation from region to region: the two spiral arms, the bar, and the satellite regions. The most striking feature is the enhancement of the SFE in regions surrounding the giant supershell. The SFE in the southern spiral arm is higher than that in the northern spiral arm. In addition, among the three satellite regions, the SFE shows high values in the two regions adjacent to the giant supershell.

What causes the enhanced SFE in regions around the giant supershell? In these regions, the PAHs/BGs abundance is also

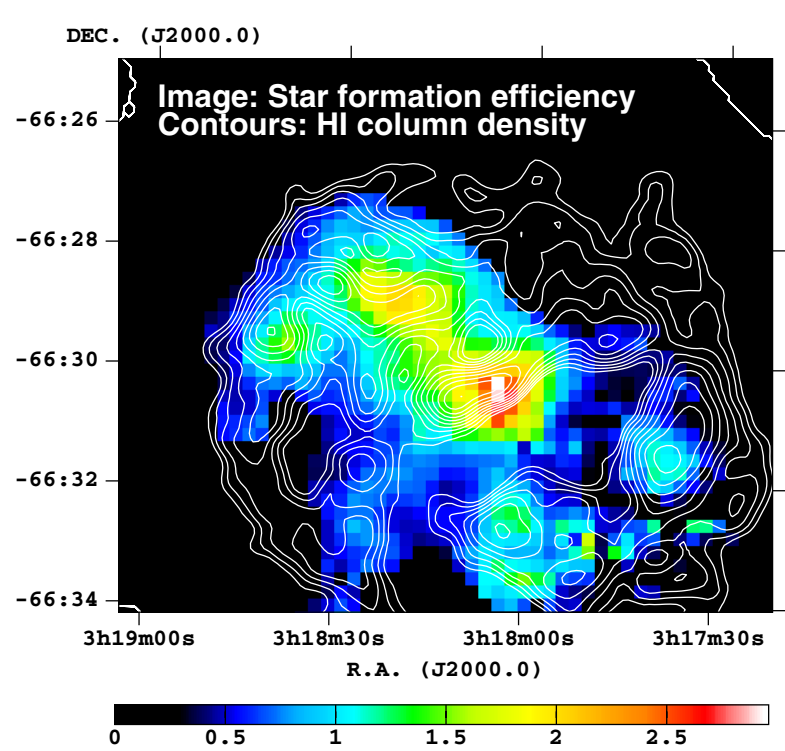

Fig. 16. Spatial distribution of SFE superposed on HI contours that are the same as those in Fig. 7. The color bar scale is in units of $10^{-9} \mathrm{yr}^{-1}$.

enhanced, as shown in Fig. 7. Considering that PAHs are associated with the cold dust and thus molecular clouds, possible mechanisms for the enhanced PAH abundance are (1) the synthesis of PAHs in molecular clouds (Greenberg et al. 2000), or (2) BG shattering and/or swept up into shells by slow shocks with velocities lower than $\sim 75 \mathrm{~km} \mathrm{~s}^{-1}$ (Jones et al. 1996; Hunter et al. 2006). Sandstrom et al. (2010) found that the PAH abundance was correlated with cold dust and $\mathrm{CO}$ emission, and suggested the possibility of PAH formation in molecular clouds. If the enhanced PAH abundance in the southern spiral arm dominantly comes from the first possibility, the cold dust luminosity in the southern spiral arm should be higher than that in the northern spiral arm. However, since these are almost the same, as shown in Fig. 5d, the first possibility is considered to be unlikely. As for the second possibility, the large-scale enhancement in the PAH abundance can be caused by the giant supershell. Since the shock velocity of the giant supershell is expected to be $\sim 40 \mathrm{~km} \mathrm{~s}^{-1}$ (Ryder et al. 1995), a possible scenario of the enhanced PAH abundance is considered to be the PAH processing and/or the PAHs swept up into the shell by the slow shock.

Furthermore, the power-law indices for the southern spiral arm and the giant supershell $(N \sim 1.6)$ are significantly different from those for the northern spiral arm and others $(N \simeq 2.0)$. The difference in $N$ is attributed to the difference in the starforming process on a kiloparsec scale. Swept-up H I gas in an expanding shell can become gravitationally unstable to form clouds and stars along the periphery: the collect-and-collapse scenario (Elmegreen \& Lada 1977). As seen in Fig. 6b, a steep ridge of $\mathrm{H}$ I underlying the southern spiral arm and satellite regions suggests that a large amount of $\mathrm{HI}$ is swept-up by the giant supershell. Once stars form, the average SFR density becomes proportional to $\omega \rho_{\text {gas }}$, where $\omega$ and $\rho_{\text {gas }}$ are the growth rate $\left(\mathrm{s}^{-1}\right)$ of an instability, which is related to the inverse of the star formation timescale and volume density of gas, respectively. Since the growth rate of gravitational instability in expanding shells is proportional to $\rho_{\text {gas }}^{0.5}$ (Elmegreen 1994a; Elmegreen et al. 2002), the SFR density is proportional to $\rho_{\text {gas }}^{1.5}$. Although the scale height of the disk seems to increase with galactocentric radius on a large scale ( $\sim 1 \mathrm{kpc}$ at 4 arcmin radius, Ryder et al. 1995), 
Table 5. Fitting parameters and their $1 \sigma$ uncertainties for the regions in NGC 1313.

\begin{tabular}{lcccc}
\hline \hline Region & $N$ & $A$ & $\epsilon$ & $\chi^{2} /$ d.o.f. \\
\hline Northern spiral arm & $2.2 \pm 0.3$ & $\left(8_{-1}^{+10}\right) \times 10^{-5}$ & $(9 \pm 3) \times 10^{-2}$ & $36.5 / 102$ \\
Southern spiral arm & $1.6 \pm 0.1$ & $(5 \pm 1) \times 10^{-4}$ & $(8 \pm 3) \times 10^{-2}$ & $48.7 / 109$ \\
Giant supershell & $1.62 \pm 0.06$ & $(4.1 \pm 0.7) \times 10^{-4}$ & $(8 \pm 3) \times 10^{-2}$ & $51.7 / 113$ \\
Others & $2.1 \pm 0.2$ & $(1.3 \pm 0.4) \times 10^{-4}$ & $(11 \pm 3) \times 10^{-2}$ & $56.5 / 115$ \\
\hline
\end{tabular}

with the assumption that the scale height of the disk within the galactocentric radius of 4 arcmin is constant, the power-law in$\operatorname{dex}, N=1.62 \pm 0.06$, obtained in the giant supershell region, can account for star formation triggered by the expanding giant supershell.

Therefore, with the above observational results, the enhanced SFE in the southern spiral arm and satellite regions strongly indicates star formation associated with the giant supershell. For the first time, our results show the possibility that giant supershells have the potential to enhance star formation activity on a galactic scale.

\section{Conclusions}

NGC 1313 was observed with the IRC and FIS onboard AKARI in ten bands $(3-160 \mu \mathrm{m})$. The ten-band images show IR emission not only from the bar and spiral arms, but also from extended regions with filamentary and patchy structures over the disk. We were able to decompose far-IR emission spectrum between $3 \mu \mathrm{m}$ and $160 \mu \mathrm{m}$ into the four emission components stars, PAHs, cold dust $(\sim 20 \mathrm{~K})$, and warm dust $(\sim 60 \mathrm{~K})$. The spatial distribution of the warm dust component shows strong emission from star-forming regions, while that of the cold dust component shows relatively extended emission along the east-west direction, as can be seen in the PAH component.

The relation between $\Sigma_{\mathrm{SFR}}$ and $\Sigma_{\text {gas }}$ that are derived from the warm and cold dust component follows a power law for the the sequence of normal disks. From a region-by-region analysis, the power-law index for the northern spiral arm is $N \simeq 2.0$, which is observed on typical spiral arms, whereas the power-law indices for the southern spiral arm and giant supershell regions show $N \sim 1$.6. The spatial distribution of the SFE shows high values in the regions around the giant supershell, with the highest SFE $\left(\sim 3 \times 10^{-9} \mathrm{yr}^{-1}\right)$ in the galaxy in the southern spiral arm region. A similar trend is found in the spatial distribution of the PAH abundance relative to BGs. The enhanced PAH abundance is considered to be caused by shattering of BGs through slow shocks $\left(40 \mathrm{~km} \mathrm{~s}^{-1}\right)$ by the giant supershell. The power-law in$\operatorname{dex} N=1.62 \pm 0.06$ obtained in the giant supershell region can be accounted for by the star formation scenario with collect-andcollapse in the expanding giant supershell. These results strongly indicate that the enhanced SFE is associated with star formation triggered by the giant supershell.

Acknowledgements. We would like to thank all members of the AKARI project for their continuous help and support. The present work is based on observations with AKARI, a JAXA project with the participation of ESA. We are grateful to the AKARI data reduction team for their extensive work in developing data analysis pipelines. We gratefully acknowledge S. D. Ryder and P. A. Crowther for providing the images of $\mathrm{H}$ I column density and $\mathrm{H} \alpha$, respectively. Some of the data presented in this paper were obtained from the MAST. STScI is operated by the Association of Universities for Research in Astronomy, Inc. under NASA contract NAS5-26555. Support for MAST for non-HST data is provided by the NASA Office of Space Science via grant NNX09AF08G and by other grants and contracts.

\section{References}

Bajaja, E., Wielebinski, R., Reuter, H.-P., Harnett, J. I., \& Hummel, E. 1995, A\&AS, 114, 147

Blanc, G. A., Heiderman, A., Gebhardt, K., Evans, II, N. J., \& Adams, J. 2009, ApJ, 704, 842

Bothwell, M. S., Kennicutt, R. C., \& Lee, J. C. 2009, MNRAS, 400, 154

Bouché, N., Cresci, G., Davies, R., et al. 2007, ApJ, 671, 303

Calzetti, D., Kennicutt, R. C., Engelbracht, C. W., et al. 2007, ApJ, 666, 870

Calzetti, D., Wu, S.-Y., Hong, S., et al. 2010, ApJ, 714, 1256

Cardelli, J. A., Clayton, G. C., \& Mathis, J. S. 1989, ApJ, 345, 245

Cluver, M. E., Appleton, P. N., Boulanger, F., et al. 2010, ApJ, 710, 248

Contursi, A., Kaufman, M. J., Helou, G., et al. 2002, AJ, 124, 751

Cox, P., \& Mezger, P. G. 1989, A\&ARv, 1, 49

Daddi, E., Bournaud, F., Walter, F., et al. 2010a, ApJ, 713, 686

Daddi, E., Elbaz, D., Walter, F., et al. 2010b, ApJ, 714, L118

de Jong, T., Clegg, P. E., Rowan-Robinson, M., et al. 1984, ApJ, 278, L67

de Vaucouleurs, G. 1963, ApJ, 137, 720

Deul, E. R., \& den Hartog, R. H. 1990, A\&A, 229, 362

Dopita, M. A., Mathewson, D. S., \& Ford, V. L. 1985, ApJ, 297, 599

Draine, B. T., \& Li, A. 2007, ApJ, 657, 810

Efremov, Y. N., Elmegreen, B. G., \& Hodge, P. W. 1998, ApJ, 501, L163

Elmegreen, B. G. 1994a, ApJ, 427, 384

Elmegreen, B. G. 1994b, ApJ, 425, L73

Elmegreen, B. G., \& Lada, C. J. 1977, ApJ, 214, 725

Elmegreen, B. G., Palouš, J., \& Ehlerová, S. 2002, MNRAS, 334, 693

Fukui, Y., Onishi, T., Abe, R., et al. 1999, PASJ, 51, 751

Genzel, R., Tacconi, L. J., Gracia-Carpio, J., et al. 2010, MNRAS, 407, 2091

Greenberg, J. M., Gillette, J. S., Muñoz Caro, G. M., et al. 2000, ApJ, 531, L71

Hadfield, L. J., \& Crowther, P. A. 2007, MNRAS, 381, 418

Heiles, C. 1979, ApJ, 229, 533

Heiles, C. 1984, ApJS, 55, 585

Hunter, D. A., Elmegreen, B. G., \& Martin, E. 2006, AJ, 132, 801

Jones, A. P., Tielens, A. G. G. M., \& Hollenbach, D. J. 1996, ApJ, 469, 740

Kaneda, H., Onaka, T., Suzuki, T., Takahashi, H., \& Yamagishi, M. 2009, in

AKARI, a Light to Illuminate the Misty Universe, eds. T. Onaka, G. J. White,

T. Nakagawa, \& I. Yamamura, ASP Conf. Ser., 418, 197

Kawada, M., Baba, H., Barthel, P. D., et al. 2007, PASJ, 59, 389

Kennicutt, R. C., Jr. 1998, ApJ, 498, 541

Leroy, A. K., Bolatto, A., Gordon, K., et al. 2011, ApJ, 737, 12

Li, A., \& Draine, B. T. 2001, ApJ, 554, 778

Marcelin, M., \& Gondoin, P. 1983, A\&AS, 51, 353

Marlowe, A. T., Heckman, T. M., Wyse, R. F. G., \& Schommer, R. 1995, ApJ, 438, 563

Martin, C. L. 1998, ApJ, 506, 222

Mathis, J. S., Mezger, P. G., \& Panagia, N. 1983, A\&A, 128, 212

McCray, R., \& Kafatos, M. 1987, ApJ, 317, 190

Méndez, B., Davis, M., Moustakas, J., et al. 2002, AJ, 124, 213

Murakami, H., Baba, H., Barthel, P., et al. 2007, PASJ, 59, 369

Onaka, T., Matsuhara, H., Wada, T., et al. 2007, PASJ, 59, 401

Peters, W. L., Freeman, K. C., Forster, J. R., Manchester, R. N., \& Ables, J. G. 1994, MNRAS, 269, 1025

Ryder, S. D., Staveley-Smith, L., Malin, D., \& Walsh, W. 1995, AJ, 109, 1592

Sandstrom, K. M., Bolatto, A. D., Draine, B. T., Bot, C., \& Stanimirović, S. 2010, ApJ, 715, 701

Sauvage, M., Tuffs, R. J., \& Popescu, C. C. 2005, Space Sci. Rev., 119, 313

Shinn, J.-H., Koo, B.-C., Burton, M. G., Lee, H.-G., \& Moon, D.-S. 2009, ApJ, 693, 1883

Shirahata, M., Matsuura, S., Hasegawa, S., et al. 2009, PASJ, 61, 737

Silva-Villa, E., \& Larsen, S. S. 2011, A\&A, 529, A25

Soifer, B. T., Neugebauer, G., \& Houck, J. R. 1987, ARA\&A, 25, 187

Suzuki, T., Kaneda, H., Onaka, T., Nakagawa, T., \& Shibai, H. 2010, A\&A, 521, A48

Tacconi, L. J., Genzel, R., Neri, R., et al. 2010, Nature, 463, 781

Tenorio-Tagle, G., \& Bodenheimer, P. 1988, ARA\&A, 26, 145

Walsh, J. R., \& Roy, J.-R. 1997, MNRAS, 288, 726 